\title{
Mundos Virtuais como apoio ao Ensino e Aprendizagem de Engenharia de Software: Simulando a Técnica de Entrevista
}

\author{
Luis Henrique Carvalho Rosa - PPGCC/UFSM - lhcr@inf.ufsm.br - \\ https://orcid.org/0000-0002-5552-2776 \\ Giliane Bernardi - PPGTER/UFSM - giliane@inf.ufsm.br - \\ https://orcid.org/0000-0002-6568-8921 \\ Roseclea Duarte Medina - PPGCC/UFSM - rose@inf.ufsm.br - \\ https://orcid.org/0000-0003-0888-6961
}

\begin{abstract}
Resumo. Esse artigo apresenta o mundo virtual LevReq3D, criado para apoiar o ensino e aprendizagem de levantamento de requisitos, por meio de um ambiente de simulação do processo de entrevista, realizado com usuários representados por agentes de software do tipo chatterbots. $\mathrm{O}$ ambiente foi avaliado por estudantes da área da computação, que analisaram o mesmo sob o ponto de vista da experiência de uso e de sua percepção em relação a aprendizagem. Como principais resultados, destaca-se a facilidade de uso, regras claras, relação com os conteúdos educacionais e potencial para aprendizagem dos mesmos, sendo que os estudantes, em sua maioria, indicaram que recomendariam o LevReq3D a outros colegas.
\end{abstract}

Palavras-chave: levantamento de requisitos; mundos virtuais 3D; chatterbot.

\section{Virtual Worlds to support teaching and learning Software Engineering: simulating the interview technique}

Abstract. This paper presents the virtual world LevReq3D, developed to support teaching and learning of software requirements elicitation. The environment simulates an interview process with users represented by chatterbots. The LevReq3D was evaluated by computer science students, considering user experience and perception of learning. The results indicate ease of use, clear rules, relation with the educational contents and potential for the learning. Finally, the students pointed that they would recommend the LevReq3D to other students.

Keywords: requirements elicitation, 3D virtual worlds; chatterbot.

\section{Introdução}

A Engenharia de Software (ES), do ponto de vista acadêmico, é uma disciplina crucial, que tem como objetivo perpassar por todas as fases de desenvolvimento de um software, desde sua concepção, passando por levantamento, análise e especificação de requisitos, codificação, testes, entre outras, envolvendo aspectos associados à qualidade e gerência de projetos. Para Mendes et al. (2019), "é fundamental o sucesso no processo de aprendizagem dos conteúdos da mesma para a boa formação dos estudantes que serão futuros profissionais engenheiros de software".

Dada à sua importância, em alguns cursos da área de computação, a ES é, inclusive, subdividida e contemplada em mais de uma disciplina. No entanto, no que concerne as fases iniciais, como a etapa de levantamento de requisitos, foco desse trabalho, usualmente os conteúdos são trabalhados de forma majoritariamente teórica, com aulas expositivas, em um modelo tradicional associado a professor/transmissor e estudante/receptor passivo das informações (MARINATO e ALVES, 2019). Essa abordagem pode tornar mais complexa a apropriação dos conteúdos enquanto conhecimentos a serem apreendidos para sua utilização enquanto futuros profissionais, 
ao mesmo tempo que desmotiva os mesmos enquanto estudantes. Ainda, autores como Brito et al. (2018) e Portela et al. (2017), reforçam que os estudantes precisam, para além do conhecimento teórico, adquirir habilidades técnicas, sendo expostos a problemas encontrados no ambiente profissional, bem como treinados para que, ao saírem da universidade, possam ter adquirido conhecimentos que os preparem para projetos reais.

Considerando esse cenário, em busca de abordagens alternativas que promovam a complementação das atividades teóricas, visando, de forma concomitante, engajar e tornar a experiência de aprendizagem de ES mais atrativa aos estudantes, emergem propostas como gamificação, jogos, simulação, entre outras. Tais abordagens trazem como perspectiva pedagógica o viés das metodologias ativas, propondo novas formas de ensinar, de forma mais centrada no aluno, em que esse passa a atuar de forma mais autônoma, em situações que o colocam em meio a tomadas de decisão, em que precisa passar por ciclos de ação-reflexão-ação, aproximando-o de situações que poderá vivenciar em seu futuro profissional (LIMA et al., 2019).

Entre as propostas de ambientes interativos que podem propiciar uma abordagem mais dinâmica para trabalhar os conteúdos destaca-se na proposta apresentada nesse artigo o uso de Mundos Virtuais em três dimensões, que possibilitam interações próximas às realizadas no mundo real. Para Herpich et al. (2020), uma das vantagens do uso de Mundos Virtuais (MV) consiste na capacidade de desprender-se das restrições da sala de aula física, incentivando sentimentos de presença mesmo quando os estudantes estão geograficamente dispersos, bem como a possibilidade de simulação de situações reais ou fictícias para o ensino de técnicas e conteúdo. Pesquisas na área de MV exploram alternativas para melhorar as interações dos estudantes com os conteúdos sendo, uma dessas, a integração com avatares autônomos, controlados por software com o intuito de simular o comportamento humano. Esses agentes são utilizados para compor cenário, dando maior realismo ao ambiente, ou para realizar interações, fornecendo informações relevantes para o cumprimento de uma determinada tarefa ou atividade.

Como o processo de levantamento de requisitos é uma atividade que exige profunda interação entre indivíduos pode ser uma estratégia promissora a inserção de inteligência nesses agentes de software por meio da construção de bases de conhecimento, possibilitando assim que eles interajam de forma mais natural e a partir das ações do estudante (agentes reativos). Tal integração pode possibilitar a criação de novas formas de interação, oferecendo ao estudante a sensação de estar interagindo com um avatar humano, um usuário que pode lhe passar informações para coleta de requisitos.

Analisando o exposto, esse artigo apresenta o desenvolvimento de um Mundo Virtual que apresenta uma simulação de dois espaços profissionais: uma empresa de desenvolvimento de software, na qual o estudante atuará como engenheiro de software; e uma pizzaria, que deseja informatizar suas operações e, para isso, contrata a empresa de software. Nessa pizzaria trabalham funcionários, representados por agentes de software, que deverão interagir com o estudante, por meio de entrevistas, fornecendo informações que permitam a coleta eficiente de requisitos para o sistema solicitado.

\section{A Técnica de Entrevista no Levantamento de Requisitos de Software}

Realizar o levantamento de requisitos é uma das primeiras atividades dentro do ciclo de vida de desenvolvimento de um software, sendo que os requisitos coletados devem refletir as necessidades do cliente e usuários finais para o software, sendo vital que os mesmos sejam corretamente elicitados, pois as funcionalidades do sistema serão implementadas de acordo com a compreensão dos engenheiros de software durante essa etapa.

Dentre as técnicas utilizadas para levantamento de requisitos, a entrevista é considerada uma das mais eficientes, pois produz descrições de contexto mais detalhadas. 
De caráter investigativo, a técnica consiste em interações entre um entrevistado, que expressa suas necessidades, e um entrevistador, o engenheiro de requisitos, que, por meio de questionamentos, busca extrair requisitos do sistema. Para cumprir essa tarefa, o entrevistador deve ter habilidade de comunicação, pois a falta de domínio desta habilidade pode provocar ambiguidade dos requisitos, insegurança do entrevistado, omissão de informações e dificuldade no entendimento dos questionamentos (JACOB e DEKKERS, 2015).

O Guia de conhecimento BABOK (Business Analysis Body of Knowledge), desenvolvido para apoiar a compreensão e execução das tarefas de análise de negócios (IIBA, 2015), divide a técnica de entrevista em três fases: preparação da entrevista, condução da entrevista e seguimento pós-entrevista, com diversas atividades em casa fase. Já Carvalho e Chiossi (2001) propõem quatro fases para aplicação da técnica: identificação dos candidatos para entrevista, preparação para entrevista, condução da entrevista e finalização da entrevista. Já em Rezende (2005) encontramos propostas de algumas diretrizes para o planejamento e execução da entrevista. Por fim, com base em sua experiência obtida a partir da execução de 280 entrevistas, Hove e Anda (2005) elencam recomendações para guiar o processo. Esses quatro estudos foram considerados para projetar o ambiente apresentado nesse artigo.

No entanto, conforme brevemente mencionado na introdução, a falta de atividades práticas e, no caso específico de levantamento de requisitos, a falta das habilidades técnicas necessárias para interagir com o entrevistado em um processo de elicitação pode comprometer a qualidade do produto final. Tais dificuldades podem ser provenientes da limitada quantidade de experiência prática fornecida para os estudantes durante sua formação acadêmica (HOVE e ANDA, 2005; JACOB e DEKKERS, 2015). Dessa forma, busca-se nesse trabalho apresentar uma proposta de complementação dos conteúdos teóricos com o auxílio de uma simulação em um Mundo Virtual, para buscar trazer ao estudante experiências simuladas de contato com o ambiente profissional, bem como engajar o mesmo em atividades inovadoras e mais atrativas. Silva (2016) relata em seu trabalho os benefícios da utilização de simulações em mundos virtuais tridimensionais como forma de capacitação do estudante em uma atividade ou técnica, ambientes que serão brevemente discutidos na próxima seção, com o objetivo de destacar como agentes de software podem ser incorporados aos mesmos para promover as interações necessárias à realização de entrevistas.

\section{Mundos Virtuais 3D e Agentes de Software}

Mundos Virtuais 3D podem ser considerados ambientes virtuais imersivos colaborativos, no qual pessoas podem interagir com diferentes propósitos, profissionais, educacionais ou, até mesmo, apenas para entretenimento/lazer (BAINBRIDGE, 2010). Ao focarmos o uso de Mundos Virtuais no campo educacional, pode-se vislumbrar os mesmos como espaços nos quais os alunos podem atuar de forma isolada, ou interagindo com outros colegas e ou agentes de software, atuando sobre artefatos, e "vivendo" experiências simuladas próximas do "mundo real", dentro de uma perspectiva de aprendizagem.

Entre os elementos que auxiliam nesse sentimento de presença e de imersão dentro de um mundo virtual destaca-se o avatar, representação gráfica do usuário (SILVA, 2016), controlado por esse para se locomover pelo mundo, interagir com artefatos, assim como executar ações, dentro do que for solicitado. Entretanto, avatares também podem ser controlados por software, atuando de forma autônoma, sendo denominados NPC Non-player Character, que podem ser utilizados para diversos propósitos, como guiar os usuários dentro do mundo, realizar tarefas, interagir com outros avatares de software ou 
humanos e simular comportamento humano, sendo conhecidos também como agentes de software (SENA, 2017).

Quando esses agentes são capazes de interagir por meio de conversas inteligentes com usuários humanos, são chamados de agentes inteligentes conversacionais ou chatterbots. Os chatterbots, quando usados em ambientes educacionais, geralmente têm o papel de auxiliar os estudantes, fornecendo informações sobre o conteúdo pedagógico, ou simulando conversas (CHOWANDA, 2016). Para realizar essas interações, os chatterbots são munidos de bases de conhecimento, desenvolvidas por meio de linguagens, como, por exemplo, a AIML (Artificial Intelligence Markup Language), uma linguagem de marcação extensível, baseada em XML, criada para o desenvolvimento de chatterbots e utilizada nessa pesquisa. Dentre os exemplos de chatterbot de maior sucesso, pode-se citar o A.L.I.C.E. (Artificial Linguistic Internet Computer Entity) criado por Dr. Richard Wallace para simular conversas humanas por meio de processamento de linguagem natural. Esses agentes são providos de bases de conhecimento que geralmente são formadas apenas pelo conteúdo específico abordado no trabalho e conhecimento sobre o ambiente, porém sem um modelo que implemente interação e personalidade e assim os chatterbots desenvolvem seus diálogos de maneira impessoal e mecânica, não se adequando ao estudante.

Considerando todo o exposto, surge a proposta apresenta, de aliar MV, por suas possibilidades enquanto ambientes de simulação, e chatterbots para explorar a técnica de entrevista em processo de levantamento de requisitos de software. A próxima seção apresenta o percurso metodológico desenvolvido para a implementação da proposta.

\section{Aspectos Metodológicos}

Essa pesquisa foi delineada seguindo a abordagem DSR - Design Science Research, que busca a integração entre a pesquisa científica com o desenvolvimento de artefatos, e que, segundo Pimentel et al. (2020), vem se popularizando cada vez mais na área de Sistemas de Informação, sendo uma abordagem eficiente para o desenvolvimento de pesquisas interdisciplinares envolvendo Educação e Computação, natureza do projeto apresentado nesse artigo.

Pimentel et al. (2020) desenvolveram um modelo, denominado Modelo-DSR, que, de acordo com os autores, apresenta uma forma de "pensar-fazer as pesquisas em DSR". Esse modelo descreve como o artefato será desenvolvido para resolver um determinado problema em um contexto específico, apoiado por um conjunto de conjecturas teóricas e referenciais técnicos. Por fim, tem-se a avaliação, que envolve: critérios de verificação do artefato, que servem para que o pesquisador possa avaliar tecnicamente o artefato; critérios de aceitação, que trazem parâmetros para avaliar se o problema foi mitigado/resolvido; e, finalmente, a avaliação empírica, que busca evidenciar o potencial de aceitação junto aos usuários-alvo.

O contexto dessa proposta envolve a diversificação das estratégias de ensino e aprendizagem de levantamento de requisitos para tentar mitigar o problema da carência de atividades mais práticas nas disciplinas de Engenharia de Software, bem como tornar a experiência de aprendizagem dos conteúdos mais engajadora e atrativa aos estudantes. Para tanto, propõe-se como artefato uma Mundo Virtual 3D interativo com a presença de agentes de software, com os quais o estudante poderá interagir em uma simulação de processo de elicitação de requisitos.

Como conjecturas teóricas, que nortearam essa pesquisa e que formaram o quadro teórico do trabalho destacam-se: mundos virtuais, enquanto ambientes que possibilitam a simulação de experiências mais próximas ao ambiente profissional futuro do estudante, de forma engajadora; e agentes de software podem promover interações que V. $19 \mathrm{~N}^{\mathrm{o}} 1$, julho, 2021 DOI: https://doi.org/10.22456/1679-1916.118527 RENOTE 
simulem um processo de entrevista, na elicitação de requisitos. Para Pimentel et al. (2020), essa relação de conjecturas e quadro teórico é o que possibilita "estabelecer uma relação entre conhecimento teórico e técnico", fazendo com que uma pesquisa não se resuma apenas a um desenvolvimento técnico.

Considerando o quadro técnico que serviu de apoio para o desenvolvimento do artefato, destaca-se: para a criação do mundo virtual, a plataforma OpenSimulator ${ }^{1}$, utilizada para construção do mundo virtual, o visualizador Imprudence ${ }^{2}$, utilizado para a construção de artefatos, e a linguagem $\mathrm{LSL}^{3}$, utilizada para a programação dos scripts necessários; para a criação dos agentes de software (chatterbots), a linguagem AIML e o servidor de bots Pandorabots ${ }^{4}$. Por fim, destaca-se que para o desenvolvimento do artefato como um todo, considerando os aspectos pedagógicos e técnicos, foi utilizada como abordagem o processo ENgAGED (BATTISTELLA e VON WANGENHEIM, 2015), voltado para o desenvolvimento de jogos educacionais para o ensino de computação, que mescla características de design instrucional com design de jogos. Dessa forma, foi criada uma unidade de estudo completa, que apresenta os objetivos educacionais $\mathrm{e}$ sequenciamento didático para uso do mundo virtual, em paralelo ao desenvolvimento do próprio ambiente.

Para a avaliação empírica, procedeu-se com a aplicação do mundo criado com uma turma de 12 estudantes da área da Computação de uma Universidade Federal do RS, sendo que para a a coleta de dados de aceitação aplicou-se o questionário proposto pelo método MEEGA+, para avaliação de jogos educacionais (PETRI et al., 2017). Na sequência, a seção 5 apresenta o mundo criado, denominado LevReq3D e, na seção 6, é apresentada a avaliação e análise de resultados.

\section{Apresentando o LevReq3D}

O ambiente LevReq3D compreende um mundo virtual que simula um ambiente real encontrado por um profissional da área de Engenharia de Requisitos, o que o classifica como um jogo de simulação, pois visa possibilitar ao estudante uma aprendizagem por meio de experiência real sem as consequências do erro. Nessa simulação, o estudante assume o papel de um engenheiro de requisitos de uma empresa de software, que deve levantar os requisitos para o desenvolvimento de um sistema online para a pizzaria, em uma simulação do tipo single-player, ou seja, apenas um estudante acessa o ambiente por vez, participando da atividade.

Para a realização do processo de elicitação de requisitos, o estudante deverá utilizar os conhecimentos sobre o conteúdo, bem como sobre diretrizes para condução de uma entrevista. Conforme mencionado na seção 2, tomou-se como referência diferentes trabalhos para estruturar a simulação, assim, o processo de entrevista projetado para a simulação foi estruturado em 03 etapas, sendo que o Quadro 01 apresenta uma síntese das mesmas, descrevendo diretrizes e atividades que devem ser executadas pelo estudante para correta pontuação de cada etapa. A unidade de estudo desenvolvida prevê que o professor, primeiramente, trabalhará o conteúdo com os estudantes de forma teórica e, após, os conduzirá à simulação no mundo virtual, para que "coloquem em prática" os conhecimentos adquiridos.

Uma entrevista pode ser realizada quantas vezes forem necessárias, até que o estudante acredite ter finalizado a atividade com sucesso. Ao término propõe-se, na

\footnotetext{
${ }^{1}$ Servidor de mundos virtuais. Disponível em: $<$ http://www.opensimulator.org $>$

${ }^{2}$ Visualizador de mundos virtuais. Disponível em: $<$ https://sourceforge.net/projects/imprudence.teampurple.p/>

${ }^{3}$ Linden Scripting Language - linguagem de programação.

${ }^{4}$ Acesso em: < https://home.pandorabots.com/home.html > V. $19 \mathrm{~N}^{\mathrm{o}} 1$, julho, 2021

DOI: https://doi.org/10.22456/1679-1916.118527 
unidade de estudo criada, que o estudante elabore um diagrama de casos de uso e um diagrama de classes, representando o sistema a ser projetado, e esses sejam entregues ao professor para avaliação. Para tal, as bases de conhecimentos dos chatterbots trazem todas as informações necessárias à construção de ambos os diagramas, sendo necessário que o estudante interaja de forma adequada para obter as mesmas da forma mais completa possível. Uma proposta de solução para os diagramas de casos de uso e classes foi inserida junto à unidade de estudo e podem ser consideradas pelo professor como gabarito. A unidade de estudo pode ser vista na íntegra em Rosa (2018).

Quadro 1. Etapas da entrevista na simulação

\begin{tabular}{|c|c|c|}
\hline Etapas & Diretrizes & Atividades \\
\hline $\begin{array}{l}\text { Preparação } \\
\text { da entrevista }\end{array}$ & $\begin{array}{l}\text { - Obter conhecimento básico do } \\
\text { projeto } \\
\text { - Definir foco e ordem lógica da } \\
\text { entrevista } \\
\text { - Agendar a entrevista }\end{array}$ & $\begin{array}{l}\text { - Analisar a descrição inicial do projeto } \\
\text { - Definir o tipo de entrevista: estruturada, } \\
\text { semi-estruturada ou não estruturada } \\
\text { - Definir a estrutura lógica das perguntas } \\
\text { - Construir as perguntas }\end{array}$ \\
\hline $\begin{array}{c}\text { Condução } \\
\text { da entrevista }\end{array}$ & $\begin{array}{l}\text { - Ser cortês } \\
\text { - Agradecer a participação do } \\
\text { stakeholder no encerramento } \\
\text { - Manter o foco nos objetivos } \\
\text { - Controlar o tempo de entrevista } \\
\text { - Não utilizar expressões técnicas }\end{array}$ & $\begin{array}{l}\text { - Apresentar-se ao entrevistado } \\
\text { - Anotar cuidadosamente as informações } \\
\text { - Registrar a entrevista }\end{array}$ \\
\hline $\begin{array}{l}\text { Pós- } \\
\text { entrevista }\end{array}$ & $\begin{array}{l}\text { - Organizar e documentar as } \\
\text { informações coletadas }\end{array}$ & $\begin{array}{l}\text { - Redigir o resumo dos tópicos abordados } \\
\text { - Elaborar o documento dos requisitos } \\
\text { levantados junto ao stakeholder }\end{array}$ \\
\hline
\end{tabular}

Fonte: dos autores.

Como locais do mundo tem-se uma empresa de software, na qual o estudante trabalha, e uma pizzaria (Figura 1), na qual encontram-se o proprietário que contratou a empresa de software (cliente/usuário), assim como os funcionários da pizzaria (usuários). Esses constituem-se como chatterbots, que interagem com o estudante no decorrer dos processos de entrevista. Todos os funcionários são representados por avatares dentro do mundo virtual, no entanto, o estudante controla seu próprio avatar, enquanto os demais são NPC, controlados por software e dotados de uma base de conhecimento sobre as funcionalidades da pizzaria. No total, tem-se 03 funcionários chatterbots, que deverão ser entrevistados para obtenção dos requisitos: o gerente proprietário, o pizzaiolo e a atendente.

Figura 1. Cenários/Locais do Mundo Virtual - Empresa de Software e Pizzaria

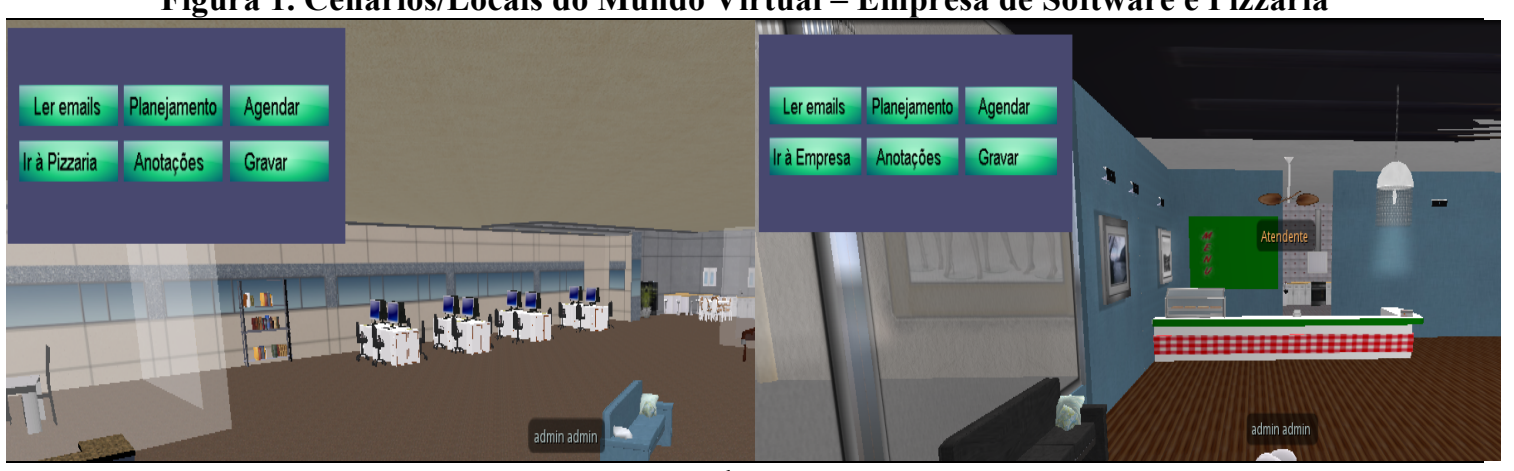

Fonte: dos autores

Ao acessar o mundo virtual, o estudante é automaticamente posicionado dentro da empresa de software, na sala do gerente de projetos (um NPC com falas pré- 
programadas), que informa que o estudante/engenheiro de requisitos tem uma tarefa, levantar todos os requisitos para o desenvolvimento de um sistema para uma pizzaria. Para isso, informa que o estudante deve-se dirigir à sua mesa de trabalho e ler o relatório inicial do projeto, que traz informações iniciais sobre a simulação, sobre a tarefa, sobre o funcionamento da área de trabalho do engenheiro, finalizando com a mensagem de que o estudante deve visitar a pizzaria para realizar as entrevistas. Tal relatório constitui o conjunto de regras e tutorial para uso do mundo virtual, dos seus artefatos e realização da simulação.

Para a execução das tarefas, o jogador contará com alguns artefatos, sendo que o principal deles é o menu do avatar, denominado Área de trabalho do Engenheiro (quadromenu na figura 01). O estudante pode executar essas ações tanto quando estiver na empresa de software, como quando se encontrar na pizzaria, com algumas limitações de ações (por exemplo, agendar uma entrevista somente pode ser feito na empresa, ele não pode ir diretamente à pizzaria e agendar um encontro já se encontrando lá). O Quadro 02 detalha cada ação possível.

Quadro 2. Área de trabalho do Engenheiro

\begin{tabular}{|l|l|}
\hline \multicolumn{1}{|c|}{ Artefato } & \multicolumn{1}{c|}{ Função } \\
\hline Ler e-mails & Essa opção abrirá a última mensagem recebida pelo engenheiro. \\
\hline Planejamento & Aqui o engenheiro poderá planejar a entrevista que irá realizar. \\
\hline Agendar & $\begin{array}{l}\text { Nessa opção o engenheiro realiza o agendamento da entrevista com um dos } \\
\text { funcionários da pizzaria. }\end{array}$ \\
\hline Anotações & $\begin{array}{l}\text { Está é uma das ferramentas disponíveis para o engenheiro, por meio dela ele } \\
\text { poderá tomar nota de informações que achar interessante. }\end{array}$ \\
\hline Gravador & $\begin{array}{l}\text { Trata-se de um gravador para que o engenheiro possa gravar toda a conversar } \\
\text { realizada com os funcionários da pizzaria. }\end{array}$ \\
\hline $\begin{array}{l}\text { Ir à Pizzaria/ } \\
\text { Ir à Empresa }\end{array}$ & $\begin{array}{l}\text { Este artefato realiza o deslocamento do engenheiro entre a empresa e } \\
\text { pizzaria, implementado por meio de uma função de teletransporte. }\end{array}$ \\
\hline
\end{tabular}
Fonte: dos autores.

Os artefatos Planejamento e Anotações habilitam um bloco de notas implementado no mundo virtual, para que o estudante possa registrar toda a preparação para a entrevista, como possíveis roteiro e perguntas a serem feitas; o artefato anotações funciona da mesma maneira, mas é habilitado na pizzaria, para que o estudante possa registrar informações da coleta e, após, consultar os dados no retorno à empresa. Todos esses registros são salvos em um banco de dados, que é acessível pelo professor para que possa ter um feedback e avaliar como se deu a organização de cada estudante no decorrer da simulação. Em decorrência do tamanho limitado para o artigo, o foco do mesmo está sendo dado à própria simulação e avaliação dos estudantes, não sendo discutidos os aspectos referentes ao painel do professor. $\mathrm{O}$ artefato Agendar permite que o estudante marque uma entrevista com um determinado usuário; caso não o faça e vá direto a pizzaria, não conseguirá interagir com o chatterbot. Por fim, destaca-se o artefato Gravador, que implementa uma função que salva, em um bloco de notas, todos os diálogos realizados entre o estudante e o agente de software (diálogo que ocorre por meio de mensagens de chat), para que o estudante possa obter a transcrição mais tarde. Cabe ressaltar que essa função somente será habilitada se o estudante questionar o entrevistado sobre essa possibilidade, pois uma das diretrizes de boa condução de entrevista é perguntar ao entrevistado se pode gravar a mesma. Na sequência, a próxima seção apresenta a avaliação e análise dos resultados da simulação apresentada. 


\section{Avaliação e Análise de Resultados}

A aplicação do LevReq3D foi realizada com 12 estudantes que, no momento da avaliação, já haviam trabalhado os conteúdos em questão na disciplina de Engenharia de Software. Assim, a unidade de estudo proposta foi adaptada, pois não houve as aulas iniciais, nas quais o professor trabalharia os conteúdos para, após, conduzir os estudantes ao jogo. $\mathrm{Na}$ ocasião da avaliação, o pesquisador, em laboratório de informática, apresentou o mundo virtual e a proposta da simulação, de forma breve, destacando que as regras e detalhes sobre a Área de Trabalho do Engenheiro seriam trazidas no próprio ambiente. Após, os estudantes realizaram a simulação e, ao final, responderam ao instrumento, que buscava analisar aspectos como usabilidade, experiência do jogador e sua percepção sobre possibilidade de aprendizagem, seguindo o questionário MEEGA+, para avaliação de jogos educacionais. A seguir, destacam-se os principais resultados, que são sintetizados nos gráficos 1 e 2 .

Para verificar se os ambientes da pizzaria e empresa foram bem construídos e trouxeram uma experiência agradável perguntou-se sobre a atratividade do design, ao que $42 \%$ dos estudantes concordam fortemente ou concordam que os ambientes se mostraram agradáveis e atraentes, enquanto $33 \%$ discordam ou discordam fortemente e $25 \%$ deles responderam de forma neutra. Isso pode evidenciar que um ambiente com maior nível de detalhes é necessário para melhorar a experiência dos estudantes no mundo virtual.

Com o propósito de verificar a clareza da dinâmica do jogo e de suas regras foi questionado se os estudantes precisaram aprender poucas coisas para começar a interagir com a simulação, sendo que $66 \%$ concordaram fortemente ou concordaram, $17 \%$ discordaram ou discordaram fortemente e $17 \%$ permaneceram neutros, o que pode significar que o ambiente apresenta uma mecânica simples e intuitiva. Quando perguntados se as regras foram claras e compreensíveis, $67 \%$ concordaram fortemente ou concordaram e os outros $33 \%$ discordaram ou discordaram fortemente, o que mostra que os propósitos e regras foram compreendidas pela maioria dos estudantes.

Ainda para entender a experiência do estudante ao jogar a simulação, foi perguntado se eles recomendariam aos colegas, ao qual $67 \%$ dos estudantes concordaram e responderam que recomendariam, já $17 \%$ discordaram e responderam que não recomendariam e $17 \%$ se mantiveram neutros, mostrando que a proposta agradou os estudantes e que pode ser utilizada para trabalhar em sala de aula.

Gráfico 1. Avaliação da experiência do Jogador

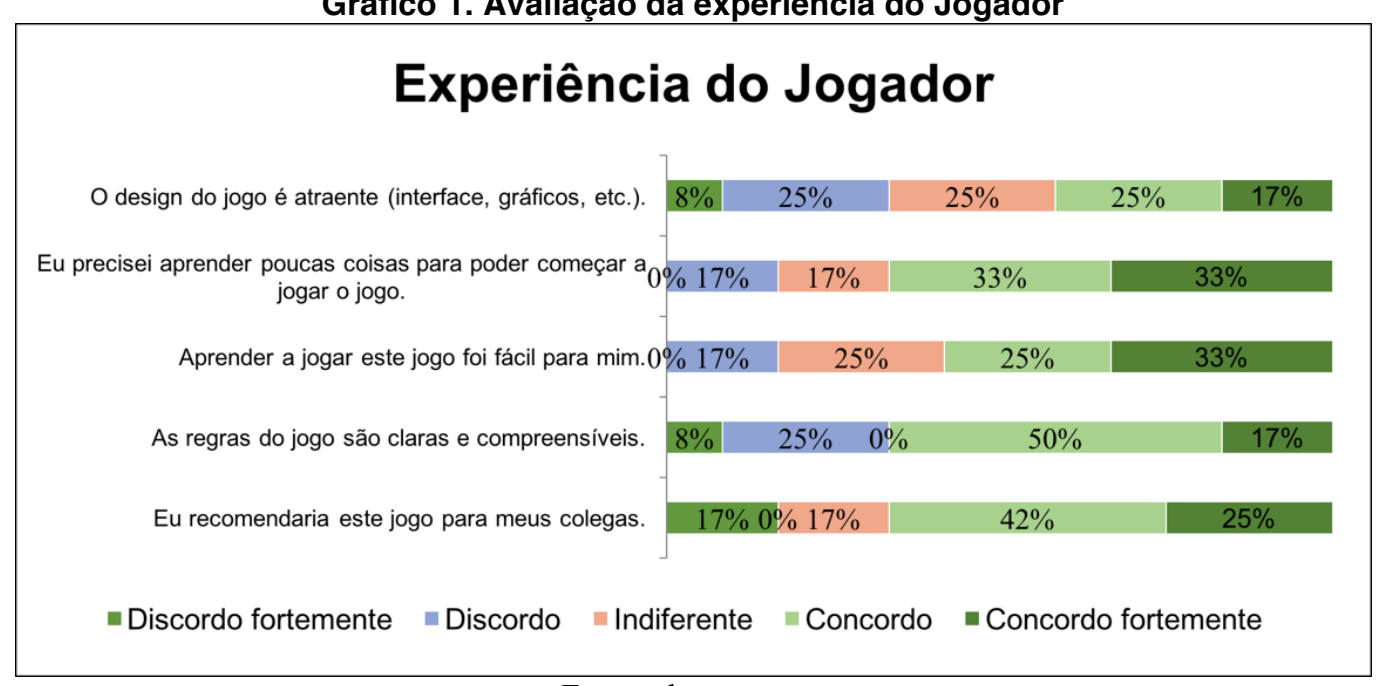

Fonte: dos autores. 
Com o propósito de verificar a percepção de aprendizagem dos estudantes (Gráfico 2), foram trazidas afirmativas aos estudantes, como: "É claro para mim como o conteúdo do jogo está relacionado ao conteúdo de Levantamento de Requisitos de Software - técnica de entrevista", sendo que 59\% dos estudantes concordaram fortemente ou concordaram com a mesma; $33 \%$ discordaram ou discordaram fortemente; enquanto $8 \%$ se mantiveram neutros. Também foi verificado se o estudante preferia aprender por meio deste jogo de simulação, por meio da afirmação "Eu prefiro aprender com este jogo do que de outra forma (outro método de ensino), neste caso 50\% dos estudantes concordaram fortemente ou concordaram, que a proposta é uma boa forma de aprender, enquanto $25 \%$ discordaram ou discordaram fortemente quanto a esse ponto e $25 \%$ responderam de forma neutra.

Por último, foi perguntado se "O jogo contribuiu para a minha aprendizagem sobre a técnica de entrevista para o Levantamento de Requisitos de Software", ao que $92 \%$ dos estudantes concordaram fortemente ou concordaram e $8 \%$ deles discordaram ou discordaram fortemente. As respostas dos estudantes indicam que o ambiente e as interações podem complementar o ensino da técnica de entrevista para o levantamento de requisitos de software.

Gráfico 2. Percepção da aprendizagem

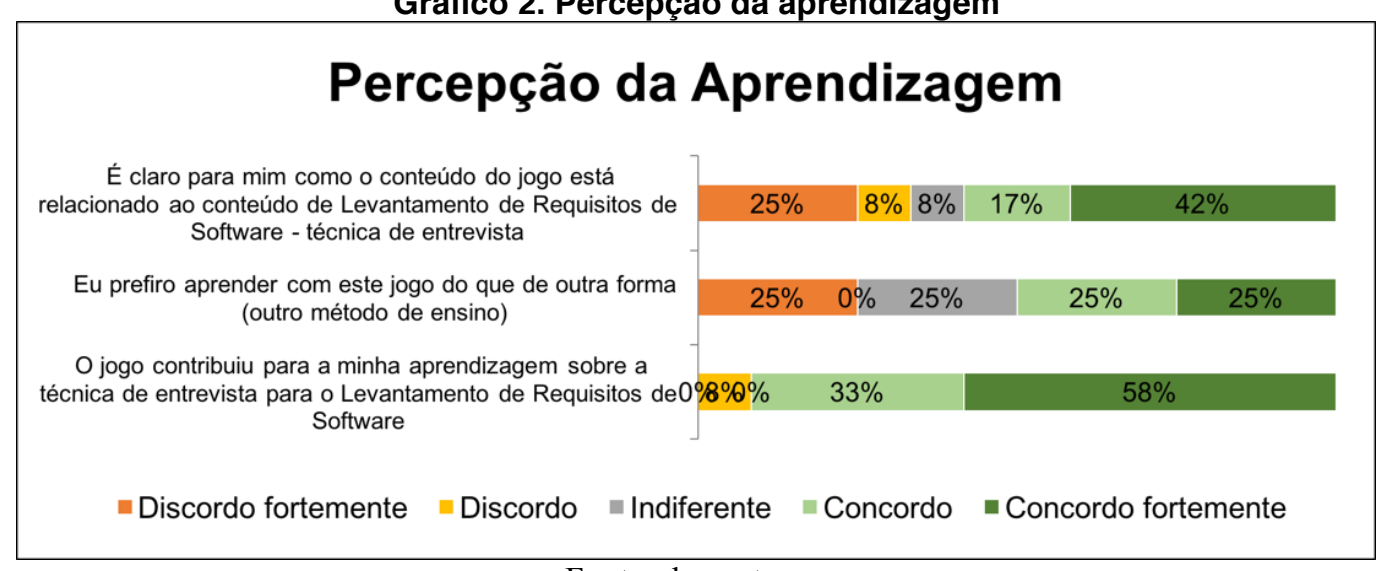

Fonte: dos autores.

A avaliação da percepção de aprendizagem em conjunto com a de experiência pode trazer indícios de que o ambiente pode proporcionar aos estudantes a experiência prática da aplicação da técnica de entrevista, tão necessária para formação de um profissional da área de levantamento de requisitos, corroborando com as afirmações de Portela et al. (2017) sobre o uso de jogos e simulação para o ensino de levantamento de requisitos, de forma lúdica e divertida, assim como o destacado por diferentes autores no que se refere às possibilidades de aliar teoria à vivências próximas da realidade por meio de Mundos Virtuais 3D (HERPICH et al., 2020; ) e promover aprendizagens mais ativas (TIBOLA e TAROUCO, 2018).

Quando avaliada a contribuição do jogo para aprendizagem, os índices mostram que o jogo pode auxiliar no processo de ensino, já no que tange a percepção do conteúdo sendo trabalhado dentro do LevReq3D, por meio do enredo, objetivos e mecânicas, estava bem estruturado, o que permitiu ao estudante a fazer relação entre os conteúdos vistos em sala de aula e as atividades desenvolvidas dentro da simulação.

\section{Considerações Finais}

Este artigo apresentou o desenvolvimento de um ambiente de simulação, imerso em um mundo virtual com o objetivo de buscar diversificar e complementar as práticas de ensino de levantamento de requisitos, em especial, a habilidade técnica de realizar entrevistas para coleta de requisitos. 
Considerando a contribuição do Mundo Virtual LevReq3D, no que concerne ao problema apresentado, é possível observar que, do ponto de vista da percepção dos estudantes, os resultados da avaliação realizada apontam para uma atividade de aprendizagem atrativa, considerando que os mesmos indicaram que recomendariam aos colegas, ao mesmo tempo que aproxima o estudante de uma experiência mais real, por meio da simulação do processo implementado. Dessa forma, as conjecturas teóricas que nortearam o projeto puderam ser confirmadas, ao constatar-se, a partir das percepções dos estudantes, que jogos do tipo simulação, assim como mundos virtuais, possibilitam experiências engajadoras e, de certa forma, realistas, próximas ao que o estudante encontrará em seu futuro profissional.

Uma fragilidade detectada foi a impossibilidade de executar a unidade de estudo de acordo com o que havia sido planejado, assim, pretende-se, como próximo estágio dessa pesquisa, aplicar novamente o ambiente junto a estudantes da disciplina de Engenharia de Software, quando os mesmos estiverem trabalhando os conteúdos referentes a levantamento de requisitos, o que ocorre, usualmente, no início do semestre. Também fica como possibilidade a implementação de outras técnicas utilizadas para o levantamento de requisitos como análise de documentos e observação. Outra modificação que pode ser realizada é do modo single-player para o modo multi-player, para que os estudantes desenvolvam a atividade em grupo, de maneira colaborativa, como o que vai ocorrer em seu ambiente profissional, na maioria das vezes, em que atuarão em equipes e ou times de desenvolvimento de software.

\section{Referências}

BAINBRIDGE, W. S. Online Worlds: Convergence of the Real and the Virtual. Human-Computer Interaction Series, Springer-Verlag, 2010.

BATTISTElla, P. E.; VON WANGENHEIM, C. G. ENgAGED: Processo de Desenvolvimento de Jogos para Ensinar Computação. Relatório Técnico do INCoD/INE, Centro Tecnológico, UFSC, 2015.

BRITO, L. F.; RAMOS, A. R.; de OLIVEIRA NETO, R. F.; LEAL, B. G. Avaliação do Jogo SIMULES no Apoio ao Ensino e Aprendizagem de Engenharia de Software. BRAJETS, v.11, n.4, 2018.

CARVALHO, A. M. B. R.; CHIOSSI, T. C. dos S. Introdução a Engenharia de Software. Sã Paulo: Editora da UNICAMP, 1a edição. 2001.

CHOWANDA, A.; BLANCHFIELD, P.; FLINTHAM, M.; VALSTAR, M. Computational models of emotion, personality, and social relationships for interactions in games. In: AAMAS 2016. 2016. p. 1343-1344.

HERPICH, F.; NUNES, F. B.; LIMA, J. V.; TAROUCO, L. M. R. Mundos Virtuais e Realidade Aumentada no âmbito educacional: reflexões e perspectivas. ReTER, 2020.

HOVE, S. E.; ANDA, B. Experiences from conducting semi-structured interviews in empirical software engineering research. In: Software metrics, 2005. IEEE. 2005.

IIBA. A Guide to the Business Analysis Body of Knowledge. V. 3.0. 2015. JACOB, N.; DEKKERS, H. Requirements Elicitation Technique: Improving the Interview Technique. 2015.

LIMA, J. V. V.; SILVA, C. A. D.; de AlEnCAR, F. M. R.; SANTOS, W. B. Metodologias Ativas como forma de reduzir os desafios do ensino em Engenharia de Software: diagnóstico de um survey. In: Anais do XXXI Simpósio Brasileiro de Informática na Educação, 2019. 
MARINATO, M, S.; ALVES, S. V. L. Uma proposta de gamificação para o ensino de Engenharia de Software. In: Anais dos Workshops do VIII Congresso Brasileiro de Informática na Educação, 2019.

MENDES, J.; COSTA, Y.; FRAZÃO, K.; SANTOS, R.; SANTOS, D.; RIVERO, L. Identificação das expectativas e dificuldades de alunos de graduação no ensino de engenharia de software. In: Anais do XXVII Workshop sobre Educação em Computação, 2019.

PETRI, G.; VON WANGENHEIM, C. G.; BORGATTO, A. F. Evolução de um Modelo de Avaliação de Jogos para o Ensino de Computação. In: 25 ${ }^{\circ}$ WEI. 2017.

PIMENTEL, M.; FILIPPO, D. SANTOS, T. M. Design Science Research: pesquisa científica atrelada ao design de artefatos. RE@D - Revista de Educação a Distância e Elearning, V.3, N.1, 2020.

PORTELA, C.; VASCONCELOS, A.; OLIVEIRA, S. R. B. Um Modelo Iterativo para o Ensino de Engenharia de Software Baseado em Abordagens Focadas no Aluno. In: Anais do Simpósio Brasileiro de Informática na Educação-SBIE, 2017.

REZENDE, D. A. Engenharia de Software e Sistemas de Informação. Brasport. 2005. SENA, S. Jogos Digitais Educativos: Design Propositions Para GDDE. Tese (Doutorado). UFSC. 2017.

ROSA, L.H.C. Jogo Sério para a Simulação da Técnica de Entrevista no Levantamento de Requisitos de Software. Dissertação (Mestrado). Programa de PósGraduação em Ciência da Computação, UFSM, 2018.

SILVA, G. J. M. A influência da comunicação não verbal na colaboração em mundos virtuais 3D: estudo de caso na formação de práticas de manutenção aeronáutica. Dissertação (Mestrado) UAB, IST. 2016.

TIBOLA, L. R.; TAROUCO, L. M. R. Laboratórios educacionais virtuais como promotores do estado de flow e da aprendizagem ativa. RENOTE, v. 16, n. 2, p. 220229, 2018. 